\begin{abstract}
We found a five-basepair insertion/deletion polymorphism in intron 3 of TNNT2, one of the genes responsible for hypertrophic cardiomyopathy. These five bases may be part of an intronic polypyrimidine tract sequence that may affect splicing. The purpose of the study was to examine the association of the polymorphism with cardiac hypertrophy. The study population consisted of 151 subjects with prominent concentric left ventricular hypertrophy, and 987 healthy subjects recruited from medical checkups (control population). The deletion/deletion genotype tended to be associated with a larger left ventricular mass/height ratio in the HCM population $(p<0.0001)$. Multiple regression analyses indicated that the left ventricular mass/height ratio was determined ( $p<0.0001, R=0.738)$ by the TNNT2geno-
\end{abstract}

The first two authors contributed equally to this study.

K. Komamura $(\bowtie)$

Department of Cardiovascular Dynamics,

Research Institute, National Cardiovascular Center,

5-7-1 Fujishirodai, Suita 565-8565, Japan

E-mail: kkoma@hsp.ncvc.go.jp

Tel.: + 81-6-6833-5012-2431

Fax: + 81-6-6872-7485

N. Iwai

Department of Epidemiology, Research Institute,

National Cardiovascular Center, Suita, Japan

K. Kokame

Department of Vascular Physiology, Research Institute,

National Cardiovascular Center, Suita, Japan

Y. Yasumura $\cdot$ J. Kim · M. Yamagishi $\cdot$ H. Tomoike

M. Kitakaze $\cdot$ K. Miyatake

Department of Cardiology, National Cardiovascular Center,

Suita, Japan

T. Morisaki

Department of Bioscience, Research Institute,

National Cardiovascular Center, Suita, Japan

\section{A. Kimura}

Department of Molecular Pathogenesis,

Medical Research Institute,

Tokyo Medical and Dental University, Tokyo, Japan type. Moreover, the frequency of the deletion allele was significantly higher in the hypertrophy population than in the control population $(p<0.0001)$. In vitro expression study revealed the deletion allele significantly affected the mRNA expression pattern by skipping exon 4 during splicing. In conclusion, TNNT2 deletion allele could be associated with a predisposition to prominent left ventricular hypertrophy.

Keywords Polymorphism - Troponin $\mathrm{T} \cdot$ Genetics · Hypertrophy $\cdot$ Cardiomyopathy

\section{Introduction}

Recent advances in molecular genetics have led to the identification of ten genes that may cause hypertrophic cardiomyopathy. These genes include the cardiac troponin $\mathrm{T}, \beta$-myosin heavy chain, cardiac myosin binding protein $\mathrm{C}, \alpha$-tropomyosin, regulatory and essential myosin light chains, cardiac troponin I, $\alpha$-actin, $\alpha$ myosin heavy chain, and titin genes (Maron 2002). All of these genes encode sarcomeric contractile proteins in the ventricle, especially in the left ventricle (LV).

We found a five-base (CTTCT) insertion/deletion (I/ D) polymorphism in intron 3 of the human cardiac troponin $\mathrm{T}$ gene (TNNT2) (Fig. 1). These $5 \mathrm{bp}$ may be part of an intronic polypyrimidine tract sequence that may affect branch site selection and splicing (Coolidge et al. 1997; Norton 1994). Intriguingly, exon 4, just distal to this polymorphism, is alternatively spliced out and removed in the cTnT2 and cTnT4 isoforms of TNNT2(Anderson et al. 1995; Mesnard et al. 1995). In addition, deletion of this exon was reported to be associated with slight reduction in myofibrillar ATPase activity (Anderson et al. 1991). Recent research indicates that the cTnT4 isoform is specifically induced in failing hearts (Anderson et al. 1995; Mesnard et al. 1995). In the present study, we examined the hypothesis that the fivebase I/D polymorphism in intron 3 of TNNT2 might 
Fig. 1 Sequences of exon 3 , intron 3, and exon 4 of TNNT2. Five-base insertions (CTTCT) are underlined. Sequence analysis of the II and DD genotypes are shown in the lower panel. Insertion allele and deletion allele are indicated in red and black, respectively
Exon 3

Intron 3

GAGCAGGAA ggtaagcgtaaacgtgtgtactcatttggatc

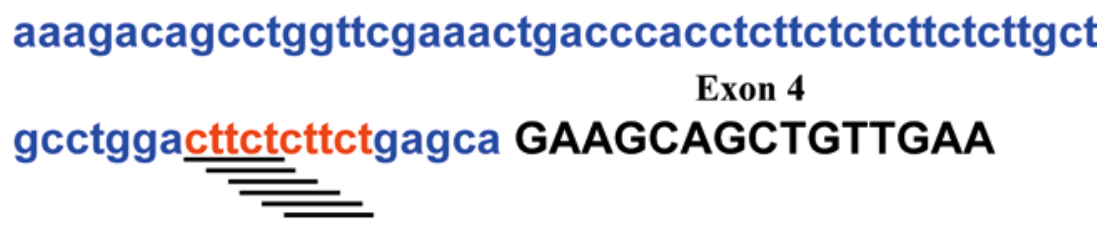

aaagacagcctggttcgaaactgacccacctcttctctcttctcttgct

Exon 4

gcctggacttctcttctgagca GAAGCAGCTGTTGAA

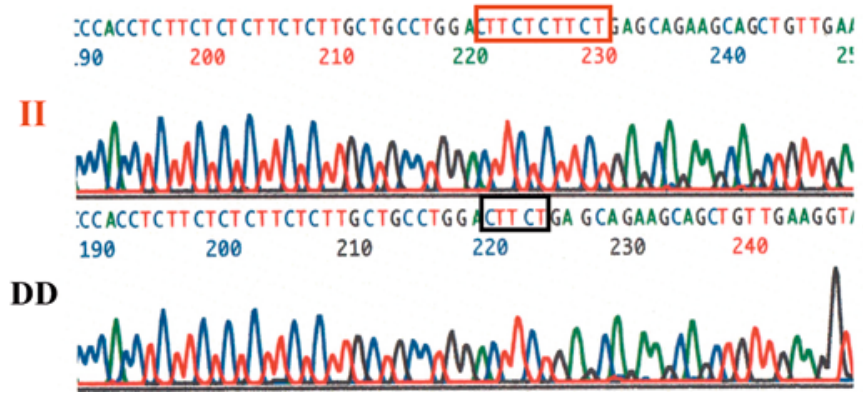

affect the skipping of exon 4, and then might affect the predisposition to LV hypertrophy.

\section{Materials and methods}

\section{Subjects}

One hundred fifty-one unrelated subjects without hypertension but with significant LV hypertrophy fulfilled the criteria based on electrocardiographic and echocardiographic measurements were recruited from the National Cardiovascular Center outpatient clinic (Hypertrophy population). Hypertension was defined as a mean systolic blood pressure of $\geq 140 \mathrm{mmHg}$, a mean diastolic blood pressure of $\geq 90 \mathrm{mmHg}$, or the patient currently taking antihypertensive medications. Strict electrocardiographic and echocardiographic criteria for the LV hypertrophy were described previously (Kuroda et al. 2001). Briefly, Cornell voltage criteria (Casale et al. 1985) and Romhilt and Estes point score system (Romhilt and Estes 1968) for electrocardiographic measurements, and a wall thickness $\geq 12 \mathrm{~mm}$ (Gardin et al. 1979), or LV mass/height was $>49.2 \mathrm{~g} / \mathrm{m}$ in men or $46.7 \mathrm{~g} / \mathrm{m}$ in women for echocardiographic measurements (Devereux et al. 1984). Screening for causative 20 mutations in TNNT2 for familial hypertrophic cardiomyopathy reported in "DNA Mutation Database for Familial Hypertrophic Cardiomyopathy" (http://www.angis.org.au/Databases/Heart/ heartbreak.html) were conducted in those subjects. We were not able to find no causative mutation in TNNT2 in those subjects.

The five-basepair I/D genotype in TNNT2 was determined using peripheral blood from the hypertrophy population and 987 healthy subjects without hypertension from the Suita Study (control population). The selection criteria and design of the Suita Study have been described previously (Iwai et al. 2001). No control subject showed any signs of significant LV hypertrophy based on the electrocardiographic criteria. The present study was approved by the Committee on Genetic Analysis and Genetic Therapy of the National Cardiovascular Center. All participants gave written informed consent.

Two-dimensionally guided M-mode measurements of LV diastolic and systolic dimensions (LVDd and LVDs), interventricular septum (IVS), and posterior wall (PW) were performed in hypertrophy population by the leading edge convention of the American Society of Echocardiography (Devereux et al. 1984). Relative wall thickness (RT) was calculated as the ratio
$\mathrm{RT}=(\mathrm{IVS}+\mathrm{PW}) / \mathrm{LVDd} \quad($ Krumholz et al. 1995). Fractional shortening (FS) was calculated as FS $=($ LVDd - LVDs $) /$ LVDd. LV mass (LVM) was calculated using a standard formula (Devereux and Reichek 1977): LVM (g) $=1.04[($ LVDd + IVS+ $\left.\mathrm{PW})^{3}-\mathrm{LVDd}^{3}\right]-13.6$. The $\mathrm{LV}$ mass index was calculated by dividing LVM by height (Luer et al. 1991).

DNA studies

DNA was isolated from peripheral leukocytes according to standard procedures. Genomic DNA from 12 subjects recruited from the National Cardiovascular Center and not included in the present study was used as a template for sequence analyses. Exons 3 and 4 of the TNNT2 gene were amplified by PCR using sense $\left(5^{\prime}\right.$ GCTACTGACAGTGTTTCCTGTTGC- $\left.3^{\prime}\right)$ and antisense (5'CCCAGGATTTCCACATTGCTGAGC-3') primers. The PCR product was gel-purified and directly sequenced using automated protocols with an ABI 310 genetic analyzer (Applied Biosystems), and used as a template for sequence analyses. The five-base (CTTCT) I/D polymorphism in intron 3 was determined by the TaqMan system (Applied Biosystems), with 5'-FamTGCCTGGACTTCTCTTCTGAGCAGAAGCAG-3' as the probe for the insertion allele and $5^{\prime}$-Tet-GCTGCCTGGACTTCTGAGCAGAAGCAGCTG-3' as the probe for the deletion allele. The results were analyzed using an ABI PRISM 7700 Sequence Detection System (Applied Biosystems) using allelic discrimination software supplied by the manufacturer.

In vitro expression study

To explore the biological significance of the five-base I/D polymorphism in intron 3, we constructed minigenes including exon 3 (9 bp), intron 3 (103 or $98 \mathrm{bp}$ ), exon 4 (15 bp), intron 4 (2,181 bp) and exon 5 (24 bp) under transcriptional control of cytomegalovirus-based promoter. Concretely, the genomic region was PCR-amplified with two primers, TNNT2-S (5'-GTGGGCAGGGCAGCGTGGACT$\left.3^{\prime}\right)$ and TNNT2-AS (5'-CTCCGTCCCTGCCGCCTACTC-3'), and inserted into the EcoRV site of a mammalian expression vector, pcDNA4/TO (Invitrogen). The direction and sequence of the inserts were confirmed by sequencing.

HeLa cells were cultured in D-MEM (Invitrogen) supplemented with 10\% FBS (Invitrogen) on 60-mm dishes in humidified air with 
$5 \% \mathrm{CO}_{2}$ at $37^{\circ} \mathrm{C}$. The subconfluent cells were transfected with $1 \mu \mathrm{g}$ of each plasmid using FuGENE6 Transfection Reagent (Roche). Following a 24-hr incubation, total RNA was isolated by a combination of Rneasy Mini Kit and Rnase-free Dnase Set (Qiagen). Then, $10 \mathrm{ng}$ of the RNA was subjected to RT-PCR using OneStep RT-PCR Kit (Qiagen). The TNNT2-S and TNNT2-AS primers $(0.6 \mu \mathrm{M}$ each) were used to amplify the spliced RNA products. The thermal cycle condition was $50^{\circ} \mathrm{C} / 30 \mathrm{~min}, 95^{\circ} \mathrm{C} / 15 \mathrm{~min}\left(94^{\circ} \mathrm{C} / 15 \mathrm{~s}\right.$, $\left.64^{\circ} \mathrm{C} / 15 \mathrm{~s}, 72^{\circ} \mathrm{C} / 30 \mathrm{~s}\right) \times 22$, and $72^{\circ} \mathrm{C} / 3 \mathrm{~min}$. The amplified cDNAs were subjected to agarose-gel electrophoresis and the bands were cut out and sequenced.

\section{Statistical analysis}

Values are expressed as the mean \pm SD. All statistical analyses were conducted using the StatView statistical software package (SAS Institute, Inc.). Stepwise multiple regression analysis was used to identify important predictors of the IVS and LVM. Age, sex, body mass index, systolic and diastolic blood pressures, pulse pressure and the $T N N T 2$ genotype $(\mathrm{II}=1, \mathrm{ID}=2, \mathrm{DD}=3)$ were included as independent predictors. Differences in numerical data among the groups were analyzed by one-way ANOVA or the unpaired $t$ test. Frequency differences among the groups were tested by contingency table analysis.

\section{Results}

Sequence analysis of TNNT2 exons 3 and 4 revealed a five-base I/D polymorphism in intron 3 (Fig. 1). The

Table 1 Characteristics of the study populations. Values are expressed as mean $\pm \mathrm{SD}$. $B M I$ Body mass index, $D B P$ diastolic blood pressure, $P P$ pulse pressure, $S B P$ systolic blood pressure

\begin{tabular}{lccl}
\hline Characteristics & Control & Hypertrophy & $P$ \\
\hline$n$ & 987 & 151 & \\
Age (years) & $58.2 \pm 12.1$ & $57.1 \pm 12.7$ & 0.2783 \\
Sex $(n, \%$ male) & $457(46.3 \%)$ & $81(53.6 \%)$ & 0.0925 \\
Height $(\mathrm{cm})$ & $159.4 \pm 8.0$ & $159.7 \pm 7.6$ & 0.7013 \\
Body weight $(\mathrm{kg})$ & $56.6 \pm 8.8$ & $57.4 \pm 8.5$ & 0.2694 \\
BMI $\left(\mathrm{kg} / \mathrm{m}^{2}\right)$ & $22.3 \pm 3.2$ & $22.6 \pm 3.5$ & 0.2575 \\
SBP $(\mathrm{mmHg})$ & $123.0 \pm 15.0$ & $122.7 \pm 14.6$ & 0.8650 \\
DBP $(\mathrm{mmHg})$ & $77.0 \pm 10.9$ & $75.5 \pm 12.0$ & 0.1254 \\
PP $(\mathrm{mmHg})$ & $46.0 \pm 10.0$ & $47.2 \pm 10.2$ & 0.1540 \\
\hline
\end{tabular}

characteristics of the study populations were summarized in Table 1. Hemodynamic background was similar between the control and hypertrophy populations. The genotype and allele frequencies in the study populations were shown in Table 2. DD or ID + DD genotype, and deletion allele might be associated with hypertrophy population. The frequencies of insertion and deletion alleles in the hypertrophy population were 33.1 and $66.9 \%$, respectively. The deletion allele frequency in the hypertrophy population was significantly higher than that in the control population $(p=0.0009)$. Effects of $\mathrm{I} / \mathrm{D}$ polymorphism on hypertrophy in the hypertrophy population were shown in Table 3. No significant effects of the TNNT2 genotype on LVDd or FS were observed. ANOVA indicated that subjects with the DD genotype had a significantly wider IVS wall thickness than subjects with other genotypes $(p<0.0001)$. Moreover, a multiple regression analysis indicated that IVS was significantly determined by the TNNT2 genotype (II $=1$, $\mathrm{ID}=2, \quad \mathrm{DD}=3, p<0.0001, r=0.738 ;$ II $+\mathrm{ID}=1$, $\mathrm{DD}=2, p<0.0001, r=0.758)$. Thus, the TNNT2deletion allele might be associated with wider IVS wall thickness.

Likewise, ANOVA indicated that subjects with the DD genotype had a significantly larger LVM/height ratio than subjects with other genotypes $(p<0.0001)$. Moreover, a multiple regression analysis indicated that $\mathrm{LVM} /$ height ratio was significantly determined by the TNNT2 genotype (II $=1, \mathrm{ID}=2, \mathrm{DD}=3, p<0.0001$, $r=0.627$; II $+\mathrm{ID}=1, \mathrm{DD}=2, p<0.0001, r=0.588$ ). Thus, the TNNT2deletion allele might be associated with increased LVM/height ratio.

We also examined the effect of the five-base I/D polymorphism in intron 3 on the TNNT2mRNA expression. After introduction of minigenes containing a part of TNNT2, ranging from the boundary of intron $2 /$ exon 3 to that of exon 5/intron 5, into HeLa cells, the spliced RNAs were detected. Different bands were observed in both insertion-allele and deletion-allele types (Fig. 2). Sequencing analysis revealed that the upper band $(320 \mathrm{bp})$ consisted of exons $3-5$ as expected,

Table 2 Genotype and allele frequencies in study populations. $D D$ deletion/deletion genotype of 5 bp in intron 3 of $T N N T 2, I D$ insertion/ deletion genotype of $5 \mathrm{bp}$ in intron 3 of $T N N T 2$, II insertion/insertion genotype of $5 \mathrm{bp}$ in intron 3 of TNNT2

\begin{tabular}{|c|c|c|c|c|}
\hline Insertion/deletion & \multicolumn{2}{|l|}{ Control } & \multicolumn{2}{|c|}{ Hypertrophy } \\
\hline II & 224 & 22.7 & 20 & 13.2 \\
\hline ID & 490 & 49.6 & 60 & 39.8 \\
\hline \multirow[t]{3}{*}{$\mathrm{DD}$} & 273 & 27.7 & 71 & 47.0 \\
\hline & II vs ID vs DD & $\chi_{2}^{2}=24.4, P=0.000005$ & & \\
\hline & II vs ID + DD & $\begin{array}{l}\chi^{2}=6.9, P=0.008412 \\
\text { odds ratio }=1.92(95 \% \text { CI, } 1.63-3.13)\end{array}$ & & \\
\hline \multicolumn{5}{|c|}{ (d) } \\
\hline Insertion & 469 & 47.5 & 50 & 33.1 \\
\hline \multirow[t]{2}{*}{ Deletion } & 518 & 52.5 & 101 & 66.9 \\
\hline & $\begin{array}{l}\chi^{2}=11.0, P=0.000933 \\
\text { Odds ratio }=1.83(95 \% \mathrm{CI}, 1.28-2.61)\end{array}$ & & & \\
\hline
\end{tabular}


Table 3 Genotypes of TNNT2 and hypertrophy. Values are expressed as mean $\pm \mathrm{SD}$. $B M I$, body mass index; $D B P$, diastolic blood pressure; $D D$, deletion/deletion genotype of $5 \mathrm{bp}$ in intron 3 of TNNT2; FS, fractional shortening; ID, deletion/deletion genotype of $5 \mathrm{bp}$ in intron 3 of TNNT2; II, insertion/insertion genotype of $5 \mathrm{bp}$ in intron 3 of TNNT2; IVS interventricular septal wall

\begin{tabular}{|c|c|c|c|c|c|c|}
\hline Characteristics & II & ID & DD & $\mathrm{II}+\mathrm{ID}$ & $P 1$ & $P 2$ \\
\hline$n$ & 20 & 60 & 71 & 80 & & \\
\hline Male/female & $7 / 13$ & $31 / 29$ & $43 / 28$ & $38 / 42$ & 0.1190 & 0.1081 \\
\hline Age (years) & $60.1 \pm 11.3$ & $58.1 \pm 13.4$ & $55.4 \pm 12.4$ & $58.6 \pm 12.8$ & 0.2575 & 0.1239 \\
\hline Height $(\mathrm{cm})$ & $158.9 \pm 6.4$ & $160.4 \pm 7.0$ & $159.3 \pm 8.5$ & $160.0 \pm 6.9$ & 0.6478 & 0.5795 \\
\hline Body weight (kg) & $58.0 \pm 8.6$ & $57.6 \pm 8.5$ & $57.1 \pm 8.5$ & $57.7 \pm 8.5$ & 0.8987 & 0.6843 \\
\hline BMI $\left(\mathrm{kg} / \mathrm{m}^{2}\right)$ & $23.1 \pm 3.6$ & $22.4 \pm 3.0$ & $22.6 \pm 3.3$ & $22.5 \pm 3.2$ & 0.7138 & 0.9747 \\
\hline $\mathrm{SBP}(\mathrm{mmHg})$ & $122.6 \pm 12.8$ & $120.2 \pm 13.5$ & $124.9 \pm 15.7$ & $120.8 \pm 13.3$ & 0.1821 & 0.0820 \\
\hline $\mathrm{DBP}(\mathrm{mmHg})$ & $77.9 \pm 9.5$ & $74.1 \pm 12.5$ & $76.1 \pm 12.2$ & $75.0 \pm 11.9$ & 0.4121 & 0.5801 \\
\hline $\mathrm{PP}(\mathrm{mmHg})$ & $44.7 \pm 5.2$ & $46.1 \pm 8.2$ & $48.8 \pm 12.3$ & $45.8 \pm 7.5$ & 0.1576 & 0.0648 \\
\hline LVDd (mm) & $49.9 \pm 5.2$ & $51.1 \pm 4.9$ & $50.1 \pm 5.2$ & $50.8 \pm 5.0$ & 0.4511 & 0.3972 \\
\hline FS $(\%)$ & $34.0 \pm 9.8$ & $34.2 \pm 6.8$ & $33.8 \pm 7.6$ & $34.1 \pm 7.6$ & 0.9547 & 0.7721 \\
\hline IVS (mm) & $13.0 \pm 0.8$ & $14.5 \pm 1.7$ & $19.6 \pm 2.9$ & $14.1 \pm 1.7$ & $<0.0001$ & $<0.0001$ \\
\hline PW (mm) & $8.2 \pm 1.0$ & $11.0 \pm 1.4$ & $12.0 \pm 2.0$ & $10.3 \pm 1.8$ & $<0.0001$ & $<0.0001$ \\
\hline RT & $0.43 \pm 0.05$ & $0.50 \pm 0.06$ & $0.64 \pm 0.09$ & $0.48 \pm 0.06$ & $<0.0001$ & $<0.0001$ \\
\hline LVM $(\mathrm{g})$ & $232.5 \pm 50.9$ & $317.9 \pm 65.0$ & $427.4 \pm 104.4$ & $296.5 \pm 71.9$ & $<0.0001$ & $<0.0001$ \\
\hline $\mathrm{LVM} /$ height $(\mathrm{g} / \mathrm{cm})$ & $1.47 \pm 0.34$ & $1.98 \pm 0.40$ & $2.70 \pm 0.71$ & $1.85 \pm 0.44$ & $<0.0001$ & $<0.0001$ \\
\hline
\end{tabular}

thickness; $L V D d$, left ventricular end-diastolic dimension; $L V M$, left ventricular mass; $P 1, P$ value obtained by separating the population into three genotypes: II, ID and DD; $P 2, P$ value obtained by separating the population into two groups: II + ID and DD; $P P$, pulse pressure; $P W$, posterior wall thickness; $R T$, relative wall thickness; $S B P$, systolic blood pressure
1998). However, the clinical significance of this polymorphism has not been elucidated. The frequency of deletion allele in the control population was over fifty percent, while not all subjects with the DD genotype in the hypertrophy population had a large LVM. Therefore, the presence of deletion allele alone did not seem to be sufficient to cause prominent cardiac hypertrophy. No subject in the hypertrophy population showed mutations in TNNT2 gene causative for familial cardiomyopathy. The deletion allele seemed to be associated with a predisposition to cardiac hypertrophy in response to various stimuli, including hemodynamic overload such as exercise, or sarcomeric dysfunction.

The five-basepair I/D site may lead to variation in the polypyrimidine tract length. This site is one of the important cis-acting sequence elements directing intron removal in mRNA splicing (Coolidge et al. 1997; Norton 1994). The $C / T$ polymorphism is in the intronic polypyrimidine tract, which has been suggested to influence splicing (Roscigno et al. 1993). Exon 4, located downstream of this polymorphism, is known to be alternatively spliced out in heart failure and in the fetal heart (Anderson et al. 1995; Mesnard et al. 1995). To date, four isoforms of human cardiac troponin $\mathrm{T}$ protein were identified in the myocardial muscle (Gomes et al. 2002). Exact data on the distribution of the isoforms in sizable number of normal hearts are not available yet. However, using a small number of samples during cardiac surgery, investigators reported that cTnT3 (missing exon 5) was the major mRNA isoform of human cardiac troponin $\mathrm{T}$, and cTnT4 (missing exons 4 and 5) was a minor isoform, while cTnT1 (all exon present) and cTnT2 (missing exon 4) mRNA were present but barely detectable (MesnardRouiller et al. 1997). Comparing to adult wild type mRNA, i.e., cTnT3, removal of exon 4 caused a slight decrease in $\mathrm{Ca}^{2+}$ sensitivity of force development in reconstituted actomyosin ATPase assay (Gomes et al. 
2002). On the contrary, others reported neither the maximal calcium-activated ATPase activity nor the calcium sensitivity was different between reconstituted myofilaments containing cTnT3 or cTnT4 (Torrealba et al. 2002). And they claimed cTnT4 might not be responsible for myocardial failure but might be a consequence of hemodynamic stress. Their finding is in agreement with the previous reports (Saba et al. 1996; Mesnard-Rouiller et al. 1997) that cTnT4 protein increased in only half the failing hearts, and correlated with the duration of inotropic support, suggesting its increase might not be associated with heart failure but might be related to hemodynamic stress. This suggestion might be consistent with our finding, i.e., the predisposition to cardiac hypertrophy.

In vitro expression study suggested that deletion polymorphism might lead to skipping of exon 4. Missing exon 4 in cardiac troponin $\mathrm{T}$ is corresponding to isoforms cTnT2 and cTnT4. Thus, deletion/deletion genotype might be associated with cTnT2 or cTnT4 mRNA isoforms in the myocardium. In the present study, we could not collect myocardial muscles from the subjects, and could not determine the troponin $\mathrm{T}$ isoforms. However, our finding suggested that deletion/deletion genotype predisposed the subjects to cardiac hypertrophy, which could be a phenotype of hemodynamic stress. The purpose of the present study was to focus on clarifying an effect of common (deletion allele percent was over fifty) insertion/deletion polymorphism on cardiac hypertrophy. In the future study, the relation between the insertion/deletion polymorphism and troponin $\mathrm{T}$ isoforms in the myocardium needs to be elucidated.

Increased LV mass is an independent predictor of cardiovascular morbidity and mortality (Kannel et al. 1972; Levy et al. 1990). Clinical features associated with cardiac hypertrophy conferred by the I/D polymorphism need further investigation. The study interpretation is limited by its small sample size. Although our findings need to be carefully interpreted, the TNNT2 deletion allele might be associated with a predisposition to prominent left ventricular hypertrophy.

Acknowledgements We are grateful to Dr. Naotaka Ohta for his technical assistance.This study was supported by a Grant-in Aid from the Ministry of Education, Culture, Sports, Science and Technology, and the Promotion of Fundamental Studies in Health Science of the Organization for Pharmaceutical Safety and Research (OPSR) of Japan.

\section{References}

Anderson PAW, Malouf NN, Oakeley AE, Pagani ED, Allen PD (1991) Troponin T isoform expression in humans. A comparison among normal and failing adult heart, fetal heart, and adult and fetal skeletal muscle. Circ Res 69:1226-1233

Anderson PA, Greig A, Mark TM, Malouf NN, Oakeley AE, Ungerleider RM, Allen PD, Kay BK (1995) Molecular basis of human cardiac troponin $\mathrm{T}$ isoforms expressed in the developing, adult, and failing heart. Circ Res 76:681-686

Casale PN, Devereux RB, Kligfield P, Eisenberg RR, Miller DH, Chaudhary BS, Phillips MC (1985) Electrocardiographic detection of left ventricular hypertrophy: development and prospective validation of improved criteria. J Am Coll Cardiol 6:572-580
Coolidge CJ, Seely RJ, Patton JG (1997) Functional analysis of the polypyrimidine tract in pre-mRNA splicing. Nucl Acids Res 225:888-896

Devereux RB, Reichek N (1977) Echocardiographic determination of left ventricular mass in men. Circulation 55:613-618

Devereux RB, Lutas EM, Cacale PN, Kligfield P, Eisenberg RR, Hammod IW, Miller DH, Reis G, Alderman MH, Laragh JH (1984) Standardization of M-mode echocardiographic left ventricular anatomic measurements. J Am Coll Cardiol 4:1222-1230

Farza H, Townsend PJ, Carrier L, Barton PJ, Mesnard L, Baehrend E, Forissier JF, Fiszman M, Yacoub MH, Schwartz K (1998) Genomic organization, alternative splicing and polymorphisms of the human cardiac troponin $\mathrm{T}$ gene. $\mathrm{J}$ Mol Cell Cardiol 30:1247-1253

Gardin JM, Henry WL, Savage DD, Ware JH, Burn C, Borer JS (1979) Echocardiographic measurements in normal subjects: evaluation of an adult population without clinically apparent heart disease. J Clin Ultrasound 7:439-447

Gomes AV, Guzman G, Zhao J, Potter JD (2002) Cardiac troponin $\mathrm{T}$ isoforms affect the $\mathrm{Ca} 2+$ sensitivity and inhibition of force development. Insights into the role of troponin T isoforms in the heart. J Biol Chem 277:35341-35349

Iwai N, Baba S, Mannami T, Katsuya T, Higaki J, Ogihara T, Ogata J (2001) Association of sodium channel $\gamma$-subunit promoter variant with blood pressure. Hypertension 38:86-89

Kannel WB, Gordon T, Castelli WP, Margolis JR (1972) Electrocardiographic left ventricular hypertrophy and risk of coronary heart disease: the Framingham Study. Ann Intern Med $72: 813-822$

Krumholz HM, Larson M, Levy D (1995) Prognosis of left ventricular geometric pattern in the Framingham Heart Study. J Am Coll Cardiol 25:879-884

Kuroda YT, Komamura K, Tatsumi R, Mori K, Yoneda K, Katayama Y, Shigemoto S, Miyatake K, Hanafusa T (2001) Vascular cell adhesion molecule-1 as a biochemical marker of left ventricular mass in the patients with hypertension. Am $\mathbf{J}$ Hypertens 14:868-872

Levy D, Garrison RJ, Savage DD, Kannel WB, Castelli WP (1990) Prognostic implications of echocardiographically determined left ventricular mass in the Framingham Heart Study. N Engl J Med 322:1561-1566

Luer MS, Anderson KM, Kannel WB, Levy D (1991) The impact of obesity on left ventricular mass and geometry. JAMA 266:231-236

Maron BJ (2002) Hypertrophic cardiomyopathy: a systemic review. JAMA 287:1308-1320

Mesnard L, Logeart D, Taviaux S, Dirlong S, Mercadier JJ, Samson F (1995) Human cardiac troponin T: cloning and expression of new isoforms in the normal and failing heart. Circ Res 76:687-692

Mesnard-Rouiller L, Mercadier JJ, Butler-Browne G, Heimburger M, Logeart D, Allen PD, Samson F (1997) Troponin T mRNA and protein isoforms in the human left ventricle: pattern of expression in failing and control hearts. $\mathbf{J}$ Mol Cell Cardiol 29:3043-3055

Norton PA (1994) Polypyrimidine tract sequences direct selection of alternative branch sites and influence protein binding. Nucl Acids Res 22:3854-3860

Romhilt DW, Estes EH (1968) A point-score system for the ECG diagnosis of left ventricular hypertrophy. Am Heart J 75:752-758

Roscigno RF, Weiner M, Garcia-Blanco M (1993) A mutational analysis of the polypyrimidine tract of introns: effects of sequence differences in pyrimidine tracts on splicing. J Biol Chem 268:11222-11229

Saba Z, Nassar R, Ungerleider RM, Oakeley AE, Anderson PA (1996) Cardiac troponin T isoform expression correlates with pathophysiological descriptors in patients who underwent corrective surgery for congenital heart disease. Circulation 94:472-476

Torrealba JR, Lozano E, Griffin M, Stoker S, McDonald K, Greaser M, Wolff MR (2002) Maximal ATPase activity and calcium sensitivity of reconstituted myofilaments are unaltered by the fetal troponin $\mathrm{T}$ re-expressed during human heart failure. J Mol Cell Cardiol 34:797-805 